\title{
ASSOCIAÇÃO ENTRE O NÍVEL DE DOR E ATIVIDADES HABITUAIS E NECESSIDADES FISIOLÓGICAS MODIFICADAS POR SUTURAS PERINEAIS DECORRENTES DE TRAUMAS NO PARTO VAGINAL
}

\author{
Maria Alice Leony de Paiva ${ }^{1}$; Luciano Marques dos Santos ${ }^{2}$ \\ 1. Bolsista PROBIC, Graduanda em Enfermagem, Universidade Estadual de Feira de Santana, e-mail: \\ alice_pitombo@hotmail.com \\ 2. Orientador, Departamento de Saúde, Universidade Estadual de Feira de Santana, Pesquisador do NUDES, e-mail: \\ lucmarxenfo@yahoo.com.br
}

PALAVRAS-CHAVE: Enfermagem Obstétrica; Dor; Períneo.

\section{INTRODUÇÃO}

A atenção às mulheres durante o processo parturitivo, há tempos vem sendo marcada por intensa medicalização. As mulheres são submetidas a rotinas e cuidados tais como o uso indiscriminado e rotineiro da episiotomia, de ocitocina intravenosa, ao jejum oral prolongado, a restrição ao leito obstétrico, estes com claros resultados negativos sobre a dinâmica do curso clínico do parto (Santos; Pereira, 2012). A prática da episiotomia e da episiorrafia, parecem ser os procedimentos que causam maiores desconfortos no pós-parto (Beleza et al., 2012).

A mulher com episiotomia tem uma experiência maior de dor e está sujeita a maior perda sanguínea, hematoma, infecção, deiscência, disfunção sexual, quando comparada com outros graus de trauma perineal (Pitangui et al., 2009). Francisco et al. (2011) trazem que, a presença da dor na mulher dificulta o exercício da maternidade, como a amamentação e o cuidado ao recém-nascido, além de interferir no desenvolvimento de atividades cotidianas, como dormir, movimentar-se, urinar, evacuar, higienizar-se e se alimentar.

O que se percebe é que há uma maior preocupação com a dor do período do parto, trabalho de parto e pós-cesárea, enquanto que a dor perineal pós-parto vaginal e pós-episiotomia são normalmente negligenciadas pelos profissionais de saúde na prática de assistência (Pitangui et al., 2009).

A partir do levantamento do estado da arte em bases de dados sobre o nível de dor associado com atividades habituais e necessidades fisiológicas modificadas nas puérperas pela presença de episiorrafia ou perineorrafia decorrente de lacerações perineais, percebeu-se a escassez de estudos tanto a nível nacional quanto internacional. Diante do exposto, questionase: há associação entre o nível de dor perineal com as atividades habituais e necessidades fisiológicas que foram modificadas nas puérperas pela presença de episiorrafia ou perineorrafia decorrente de lacerações perineais? Por isso, esse estudo justifica-se pela necessidade de caracterização da dor, decorrente da laceração e episiotomia, e suas implicações nas atividades diárias das puérperas para que o profissional de saúde possa intervir e prestar uma assistência de qualidade à essa puérpera.

Tem como objetivo geral: verificar a associação entre o nível de dor perineal com as atividades habituais e necessidades fisiológicas que foram modificadas nas puérperas pela presença de episiorrafia ou perineorrafia decorrente de lacerações perineais no parto vaginal em uma instituição pública de Feira de Santana, Bahia. Tem como objetivos específicos: mensurar o nível de dor decorrente de episiorrafia ou perineorrafia decorrente de lacerações perineais no pós-parto imediato; descrever a associação da dor com o tipo de trauma perineal decorrente do parto; analisar a associação entre o nível de dor perineal com as atividades habituais das puérperas que foram modificadas pela presença de episiorrafia ou perineorrafia decorrente de lacerações perineais. 


\section{MÉTODOS}

Trata-se de um estudo transversal, realizado no município de Feira de Santana-Bahia, no Hospital Inácia Pinto dos Santos (HIPS), na enfermaria de Alojamento Conjunto. Os dados utilizados para elaborar este estudo foram secundários do banco de dados do projeto de pesquisa "Condições perineais de mulheres no pós-parto vaginal em uma instituição pública do interior da Bahia", aprovado pelo comitê de ética em pesquisa da Universidade Estadual de Feira de Santana, através do parecer de $\mathrm{n}^{\circ} 842$. 198. As informações deste banco foram obtidas através de entrevista com aplicação de um formulário e exame físico de todas as puérperas submetidas ao parto normal na unidade em estudo, que apresentaram integridade e trauma perineal.

Os dados foram tratados e analisados por meio do pacote estatístico Statistical Package for the Social Sciences (SPSS), versão 15.0 e apresentados sob a forma de tabelas. A técnica de análise dos dados foi a estatística descritiva e inferencial. Foi realizada a contagem das frequências simples e o cruzamento das variáveis pertinentes ao estudo. No cruzamento das variáveis, foi utilizado o método de qui-quadrado e considerado como significantes os resultados com o $\mathrm{p}<0,05$. Foram calculadas as razões de prevalência (RP) e seus respectivos intervalos de confiança de $95 \%$. O presente estudo dispensou a utilização de um Termo de Consentimento Livre e Esclarecido (TCLE), já que o contato da pesquisadora foi apenas com o banco de dados do projeto de pesquisa já referido e a mesma se comprometeu em utilizar o banco de dados bem como suas informações com o cuidado necessário e respeito aos aspectos éticos.

\section{RESULTADOS}

Tabela 1 - Distribuição do nível de dor perineal em mulheres de acordo com o trauma local no pós-parto vaginal em uma instituição pública de Feira de Santana (BA). Set/2013-

$\mathrm{dez} / 2014$.

\begin{tabular}{lccc}
\hline Nível de dor & Leve $\mathbf{N}(\%)^{*}$ & Moderado $\mathbf{N}(\%)^{* * *}$ & Grave $\mathbf{N}(\%) * * *$ \\
\hline Trauma perineal & & & \\
Laceração & $94(21,9)$ & $132(30,7)$ & $204(47,4)$ \\
Episiotomia & $86(19,5)$ & $163(37)$ & $192(43,5)$ \\
Ambos & $2(20)$ & $6(60)$ & $2(20)$
\end{tabular}

FONTE: Banco de dados do Projeto de Pesquisa "Condições perineais de mulheres no pósparto vaginal em uma Instituição pública do interior da Bahia”, 2014.

*Considera-se dor leve 1 a 3 pontos na escala numérica de avaliação da dor.

*Considera-se dor moderada 4 a 7 pontos.

*Considera-se dor grave 8 a 10 pontos.

Tabela 2- Associação da ocorrência de dor com as atividades habituais modificadas pelas mulheres que tiveram parto vaginal com trauma perineal, em uma instituição pública, Feira de

Santana (BA), set/2012-dez/2014.

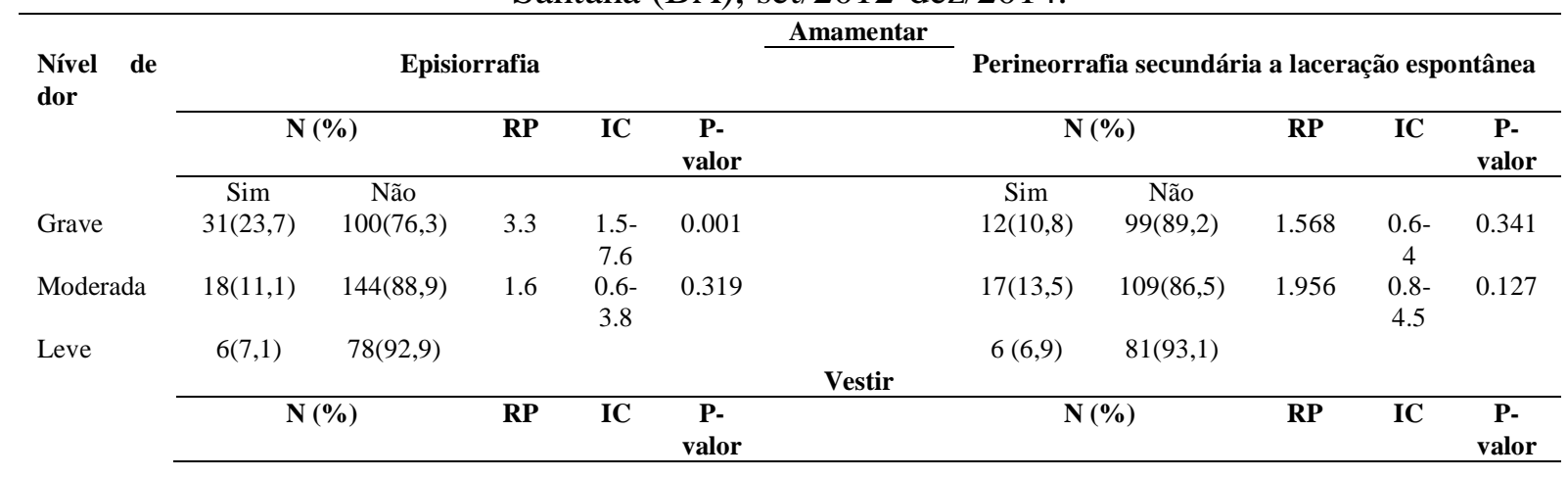




\begin{tabular}{|c|c|c|c|c|c|c|c|c|c|c|}
\hline & Sim & Não & & & & Sim & Não & & & \\
\hline Grave & $39(29,8)$ & $92(70,2)$ & 1.786 & $1-3$ & 0.029 & $21(18,9)$ & $90(81,1)$ & 1.11 & $\begin{array}{c}0.6- \\
2\end{array}$ & 0.733 \\
\hline Moderada & $32(19,8)$ & $130(80,2)$ & 1.185 & $\begin{array}{c}0.7- \\
2\end{array}$ & 0.556 & $17(13,5)$ & $109(86,5)$ & 0.7915 & $\begin{array}{c}0.4- \\
1.5\end{array}$ & 0.473 \\
\hline \multirow[t]{4}{*}{ Leve } & $14(16,7)$ & $70(83,3)$ & & & & $15(17)$ & $73(83)$ & & & \\
\hline & \multicolumn{10}{|c|}{ Deambular } \\
\hline & \multicolumn{2}{|c|}{$\mathbf{N}(\%)$} & $\mathbf{R P}$ & IC & $\begin{array}{c}\text { P- } \\
\text { valor }\end{array}$ & \multicolumn{2}{|c|}{$\mathbf{N}(\%)$} & $\mathbf{R P}$ & IC & $\begin{array}{c}\text { P- } \\
\text { valor }\end{array}$ \\
\hline & $\mathrm{Sim}$ & Não & & & & Sim & Não & & & \\
\hline Grave & $92(70,2)$ & $39(29,8)$ & 1.84 & $\begin{array}{c}1.4- \\
2.5\end{array}$ & 0.000 & $48(43,2)$ & $63(56,8)$ & 1.028 & $\begin{array}{c}0.7- \\
1.4\end{array}$ & 0.865 \\
\hline Moderada & $108(66,7)$ & $54(33,3)$ & 1.75 & $\begin{array}{l}1.3- \\
2.4\end{array}$ & 0.000 & $76(60,3)$ & $50(39,7)$ & 1.435 & $\begin{array}{c}1- \\
1.9\end{array}$ & 0.008 \\
\hline \multirow[t]{3}{*}{ Leve } & $32(38,1)$ & $52(61,9)$ & & & & $37(42)$ & $51(58)$ & & & \\
\hline & \multicolumn{10}{|c|}{ Sentar } \\
\hline & \multicolumn{2}{|c|}{$\mathbf{N}(\%)$} & $\mathbf{R P}$ & IC & $\begin{array}{c}\text { P- } \\
\text { valor }\end{array}$ & \multicolumn{2}{|c|}{$\mathbf{N}(\%)$} & $\mathbf{R P}$ & IC & $\begin{array}{c}\text { P- } \\
\text { valor }\end{array}$ \\
\hline Grave & $\begin{array}{c}\text { Sim } \\
114(85,9)\end{array}$ & $\begin{array}{c}\text { Não } \\
53(14,1)\end{array}$ & 0.88 & $\begin{array}{c}0.7- \\
1\end{array}$ & 0.131 & $\begin{array}{c}\text { Sim } \\
77(68,8)\end{array}$ & $\begin{array}{c}\text { Não } \\
35(31,3)\end{array}$ & 0.864 & $\begin{array}{c}0.7- \\
1\end{array}$ & 0.085 \\
\hline Moderada & $145(89,5)$ & $17(10,5)$ & 1.15 & $\begin{array}{c}1- \\
1.3\end{array}$ & 0.010 & $112(88,9)$ & $14(11,1)$ & 1.117 & $\begin{array}{c}0.9- \\
1.2\end{array}$ & 0.059 \\
\hline Leve & $65(77,4)$ & $19(22,6)$ & & & & $70(79,5)$ & $18(20,5)$ & & & \\
\hline
\end{tabular}

FONTE: Banco de dados do Projeto de Pesquisa "Condições perineais de mulheres no pósparto vaginal em uma Instituição pública do interior da Bahia”, 2014.

Tabela 3- Associação da ocorrência de dor com as necessidades fisiológicas modificadas pelas mulheres que tiveram parto vaginal com trauma perineal, em uma instituição pública, Feira de Santana (BA), set/2012-dez/2014.

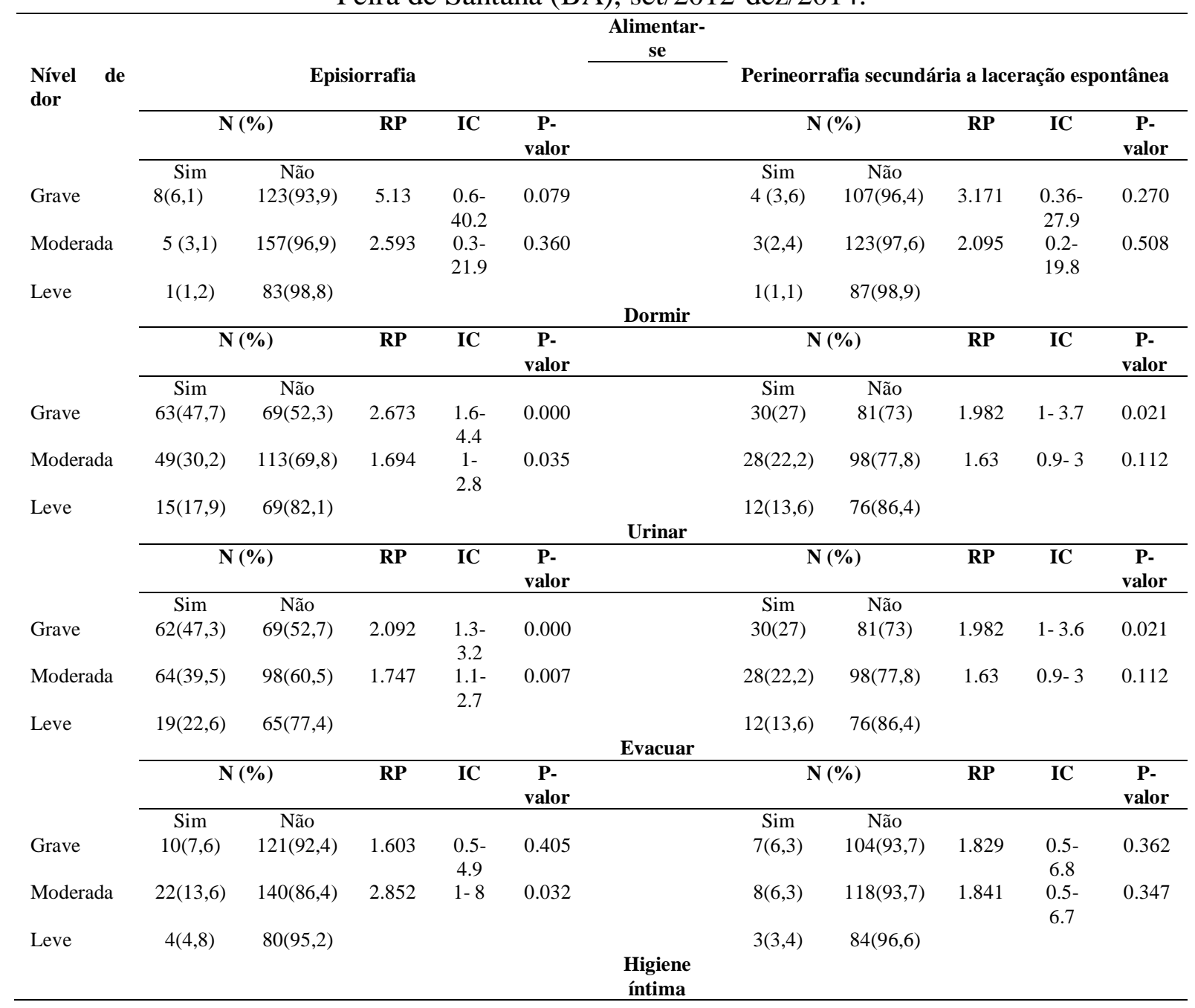




\begin{tabular}{|c|c|c|c|c|c|c|c|c|c|c|}
\hline \multirow[b]{2}{*}{ Grave } & \multicolumn{2}{|c|}{$\mathbf{N}(\%)$} & \multirow{2}{*}{$\begin{array}{c}\mathbf{R P} \\
2.188\end{array}$} & \multirow{2}{*}{$\begin{array}{l}\text { IC } \\
1.3- \\
3.5\end{array}$} & \multirow{2}{*}{$\begin{array}{c}\begin{array}{c}\text { P- } \\
\text { valor }\end{array} \\
0.000\end{array}$} & \multicolumn{2}{|c|}{$\mathbf{N}(\%)$} & \multirow{2}{*}{$\begin{array}{c}\mathbf{R P} \\
1.306\end{array}$} & \multirow{2}{*}{$\begin{array}{c}\text { IC } \\
0.9- \\
1.8\end{array}$} & \multirow{2}{*}{$\begin{array}{c}\begin{array}{c}\text { P- } \\
\text { valor }\end{array} \\
0.095\end{array}$} \\
\hline & $\begin{array}{c}\mathrm{Sim} \\
58(44,3)\end{array}$ & $\begin{array}{c}\text { Não } \\
73(55,7)\end{array}$ & & & & $\begin{array}{c}\mathrm{Sim} \\
57(50,4)\end{array}$ & $\begin{array}{c}\text { Não } \\
56(49,6)\end{array}$ & & & \\
\hline Moderada & $54(33,3)$ & $108(66,7)$ & 1.647 & $\begin{array}{c}1- \\
2.6\end{array}$ & 0.031 & $61(48,4)$ & $65(51,6)$ & 1.253 & $\begin{array}{l}0.9- \\
1.7\end{array}$ & 0.156 \\
\hline Leve & $17(20,2)$ & $67(79,8)$ & & & & $34(38,6)$ & $54(61,4)$ & & & \\
\hline
\end{tabular}

FONTE: Banco de dados do Projeto de Pesquisa "Condições perineais de mulheres no pósparto vaginal em uma Instituição pública do interior da Bahia”, 2014.

Foram estudadas 881 mulheres. As mulheres com laceração e episiotomia queixam-se mais de dor perineal grave $(47,4 \%$ e $43,5 \%$ respectivamente), seguida do nível moderado (30,7\% e $37 \%$ respectivamente). Nas associações estatisticamente significantes mulheres com dor decorrente da episiorrafia relataram maior dificuldade para amamentar $(\mathrm{p}=0,001 ; \mathrm{RP}=3.3$; $\mathrm{IC}=1.5-7.6)$, dormir ( $\mathrm{p}=0,000 ; \mathrm{RP}=2.673$; $\mathrm{IC}=1.6-4.4)$, vestir-se $(\mathrm{p}=0,029 ; \mathrm{RP}=1.786$; $\mathrm{IC}=1$ $3)$, deambular $(\mathrm{p}=0,000 ; \mathrm{RP}=1.84 ; \mathrm{IC}=1.4-2.5)$, higiene íntima $(\mathrm{p}=0,000 ; \mathrm{RP}=2.188 ; \mathrm{IC}=1.3$ 3.5); e urinar $(\mathrm{p}=0,007 ; \mathrm{RP}=2.092 ; \mathrm{IC}: 1.3-3.2)$. Mulheres com dor grave decorrente de perineorrafia secundária a laceração espontânea apresentaram maior dificuldade para urinar $(\mathrm{p}=$ 0,021)

Francisco et al. (2012) demonstraram que a presença de episiorrafia acarretou a dor, e esta consequentemente dificultou as atividades como sentar, com 90,4\% e 75\%, andar com $48,1 \%$ e $53,8 \%$ e dormir com $38,5 \%$ e $36,5 \%$ das entrevistadas, com 20-24 horas e 40-48 horas após o nascimento, respectivamente.

Beleza et al. (2012), com cinquenta puérperas que referiram dor no pós-parto após a episiotomia constatou que $52 \%$ delas informaram sentir dor ainda em repouso. Com relação as atividades habituais modificadas pela dor foram referidas: sentar $(49,8 \%)$, deambular $(41,81 \%)$, higiene íntima $(22,44 \%)$, deitar $(45,90 \%)$, evacuar $(2,4 \%)$, micção $(20,40 \%)$ e sono $(18,36 \%)$. Estes dados reforçam que a dor é um fator limitante para que as mulheres exerçam suas atividades e promovam o autocuidado e cuidado com recém-nascido.

\section{CONCLUSÃO}

Os resultados mostram a importância da avaliação da dor perineal no pós-parto imediato pela equipe profissional, que tem a responsabilidade de valorizar a queixa dolorosa e avaliá-la não somente unidimensionalmente, mas em intensidade e caráter, buscando formas de atenuar as mudanças nas atividades diárias das puérperas, no cuidado de si e do seu recém-nascido.

\section{REFERÊNCIAS}

BELEZA, A.C.S et al. 2012. [online]. Mensuração e caracterização da dor após episiotomia e sua relação com a limitação de atividades. Rev bras enferm Brasília 65(2): 264-8. Homepage: < http://www.scielo.br/pdf/reben/v65n2/v65n2a10.pdf >

FRANCISCO, A.A et al. 2011. [online]. Avaliação e tratamento da dor perineal no pós-parto vaginal. Acta paul enferm. 24(1):94-100. Homepage < http://www.scielo.br/pdf/ape/v24n1/v24n1a14.pdf $>$

FRANCISCO AA, et al. 2012. [online]. Efecto del dolor perineal em las actividades de mujeres sometidas a episiotomía. Index enferm Granada. 21(3):150-4. Homepage < http://scielo.isciii.es/scielo.php?script=sci_arttext\&pid=S1132-12962012000200009> PITANGUI, A.C.R et al. 2009. [online]. Measurement and characteristics of perineal pain in primiparous undergoing episiotomy. Acta paul enferm. 22 (1): 77-82. Homepage < http://www.scielo.br/scielo.php?script=sci_arttext\&pid=S0103-21002009000100013> SANTOS, L.M; PEREIRA, S.S.C. 2012. [online]. Vivências de mulheres sobre a assistência recebida no processo parturitivo. Physis Rev Saúde Coletiva. 22 (1): 77-97. Homepage: < http://www.scielo.br/scielo.php?script=sci_arttext\&pid=S0103-73312012000100005> 